International Journal of Robotics and Automation (IJRA)

Vol. 10, No. 4, December 2021, pp. 340 352

ISSN: 2089-4856, DOI: 10.11591/ijra.v10i4.pp340-352

\title{
Low-cost SARS-CoV-2 vaccine homogenization system for Pfizer-BioNTech covid-19 vials
}

\author{
José Lima ${ }^{1}$, Luísa Rocha ${ }^{2}$, Cláudia Rocha ${ }^{3}$, Paulo Costa ${ }^{4}$ \\ ${ }^{1}$ Research Centre of Digitalization and Intelligent Robotics, Instituto Politécnico de Bragança, Braganca, Portugal \\ ${ }^{2}$ Hospital Pharmacy, Centro Hospitalar de Vila Nova de Gaia/Espinho, E.P.E. (CHVNG/E, E.P.E.), Portugal \\ ${ }^{1,3,4}$ INESC Technology and Science, Porto, Portugal \\ ${ }^{4}$ Faculty of Engineering of University of Porto, Porto, Portugal
}

\section{Article Info}

Article history:

Received Apr 2, 2021

Revised Jul 29, 2021

Accepted Aug 19, 2021

\section{Keywords:}

Covid-19

Homogenization

Pharmacy preparations and

compounding

SARS-CoV-2 vaccine

\begin{abstract}
The current SARS-CoV-2 pandemic has been affecting all sectors worldwide, and efforts have been targeting the enhancement of people's health and labour conditions of collaborators belonging to healthcare institutions. The recent vaccines emerging against covid-19 are seen as a solution to address the problem that has already killed up to two million people. The preparation of the Pfizer-BioNTech covid-19 vaccine requires a specific manipulation before its administration. A correct homogenization with saline solution is needed and, therefore, a manual process with a predefined protocol should be accomplished. This action can endanger the operators' ergonomics due to the repetitive movement of the process. This paper proposes a low-cost prototype incorporating an arduino based embedded system actuating a servomotor to perform an autonomous vials' homogenization allowing to redirect these healthcare workers to other tasks. Moreover, a contactless start order process was implemented to avoid contact with the operator and, consequently, the contamination. The prototype was successfully tested and recognised, and is being applied during the preparation of the covid-19 vaccines at the hospital pharmacy of Centro Hospitalar de Vila Nova de Gaia/Espinho, E.P.E., Portugal. It can be easily replicated since the source files to assemble it are provided by the authors.
\end{abstract}

This is an open access article under the CC BY-SA license.

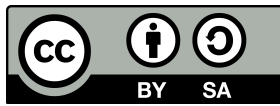

\section{Corresponding Author:}

José Lima,

Research Centre of Digitalization and Intelligent Robotics

Instituto Politécnico de Bragança,

INESC Technology and Science, Porto, Portugal

Email: jllima@ipb.pt

\section{INTRODUCTION}

Coronavirus disease 2019, colloquially known as covid-19, is an infectious disease caused by a kind of virus, designated as coronavirus - SARS-CoV-2, and it was primarily identified in humans in the end of 2019 [1]. It is considered a respiratory syndrome that can cause mild to severe symptoms. According to the world health organization (WHO), more than two millions of people have died due to complications caused by the covid-19 infection since December 2019 [2]. This number is increasing day after day and, in accordance with recent forecasts, the predictions are not encouraging [3]. Several measures have been applied namely confinement or social withdrawal but the numbers of transmission between humans are not decreasing. Recently, diverse pharmaceutical laboratories presented vaccines in an attempt to eradicate this global pandemic. 
To avoid pandemic propagation, the viral transmission (reproduction number $R o$ ) must remain below 1 , meaning that each infected person transmits on average less than one additional individual. This is a key aim for continuously reducing case numbers. In contrast, a reproduction number $R o$ greater than 1 means a highly unfavourable exponential increase of new infections [5]. So, the main concern is to perform the vaccination process as fast as possible. According to [6], more than 200 covid-19 vaccines are in development worldwide, with governments securing deals to have early access to a large number of doses. Nonetheless, access is only one issue, since there are other obstacles. The willingness to accept a covid-19 vaccine when it becomes available has varied considerably across countries over the course of the pandemic; the storage; the logistics; the preparation of the vaccines; and the administration are issues that should be overcome [6]. Pfizer ${ }^{\mathrm{TM}}$ made the announcement on 9 November 2020 and offered the first compelling evidence that a vaccine can prevent covid-19 [7].

The vaccination process has been started in several countries with the priority of the older people and health professionals, such as doctors, nurses, pharmacists and technicians, among others. The preparation of the vaccines depends on the respective vaccine summary of product characteristics (SmPC). For the PfizerBioNTech covid-19 vaccine, presented on Figure 1 (which preliminary findings suggest efficacy of the vaccine to be as high as $95 \%$ [4]), handling is required during three main phases: the homogenization, the dilution, and a second homogenization. The dilution process consists of adding saline to the vial whereas the homogenization process requires the pharmacists to carefully manipulate the vial several times, a repetitive procedure that can lead to wrist injuries.

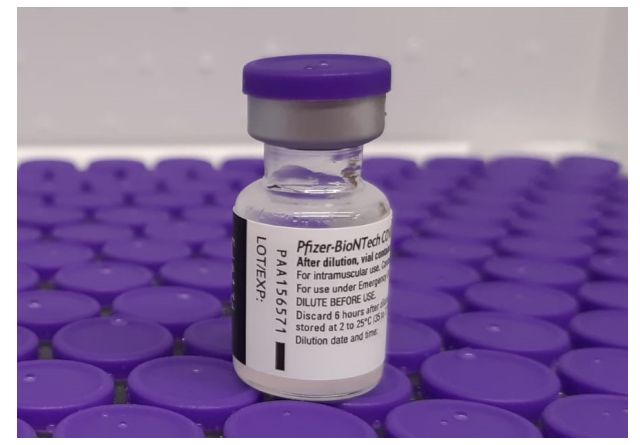

Figure 1. A vial of Pfizer-BioNTech covid-19 vaccine

In the pre-covid and initial pandemic phases, the autonomous homogenization of vaccines was not necessary as the vaccination was an occasional procedure and did not pose a threat for the ergonomics of the healthcare personnel. However, we are now facing a time where the quick and widespread vaccination is of utmost importance. Therefore, manual homogenization is now becoming a very repetitive task, posing health risks. The massive vaccination all around the world, due to covid-19, requires an infrastructure capable of supporting the preparation and administration for all the people. The governments and vaccine developers are trying their best to introduce the vaccines for general public use [8]. It brought some problems that did not exist before: pharmaceutical and laboratory companies were pressured to develop a vaccine; logistics and storage issues due to the hard conditions like low temperatures (in some cases); the preparation involving the manipulation of the vials and dilution should be carefully done; and, finally, the administration on the world population.

Homogenization is a widely used process in laboratories and poses health risks due to repetitive stress to the wrist joint when manually performed. Therefore, innovation has focused its automation so that the operator can avoid complications and be directed to non-repetitive specific tasks. The existing automated homogenization systems are not suitable for every type of sample and/or container, so efforts have been made to improve laboratory methods. Different homogenization methods for elastic collagen-rich tissues are analysed in [9]. The authors proposed a novel microtome-cryostat apparatus which is adequate to the requirements of those materials, as opposed to methods such as vortexer bead beating, glass homogenizer and rotor homogenizer. This approach uses a cryotome and is a complex solution when compared to the one proposed on this paper. In another work, a fully automated ultrasonic robot was developed for the homogenization of filamentous fungal cells [10]. It comprises a vision system to distinguish empty from sample-containing wells, and also 
assesses the level of fungi homogeneity. The authors have extended their system by adding centrifuge and liquid handling modules, in order to automate the preparation of fungal samples too [11]. It is also a complex solution but interesting for other purposes such as splitting components of a fluid with different densities. For our particular scenario, there have been proposed several commercial products such as rotating mixers, roller mixers, agitators, among others; however, none of them is capable of performing the 180 degrees turn on the clockwise and counter clockwise directions, as specified in the summary of product characteristics (SmPC), a procedure that must be followed during the homogenization process. Some examples are the Partner Rotamix ${ }^{\mathrm{TM}}$ from the electron microscopy sciences manufacturer [12], the mini LabRoller rotator with Rotisserie from the Labnet $^{\mathrm{TM}}$ manufacturer [13], and the rotating mixer from benchmark scientific [14], all of which continuously turn in the same direction. Other examples are the blood mixer from meditech [15] and the MediMix Vial reconstitution mixer from pharma systems [16], both of which perform a circular movement different than the desired one, and the tube rocker from Unico [17] acting as an agitator. These similar commercial products were stressed but none of them provides all the features of the proposed model: low cost, easily replicable and the capability of performing the movement specified in the SmPC.

Naturally, the homogenization process can be done manually. The problem is that whenever an articulation in the body is subjected to repetitive movements, there is a risk of developing musculoskeletal disorders. These comprise injuries of the muscles, nerves, tendons, joints, cartilage, and spinal discs, such as sprains, carpal tunnel syndrome, hernias, pain, among others, which can persist or even aggravate when having an inadequate posture during labour or due to repetitive tasks [18]. In the particular case of the manual homogenization process, torsional forces are applied on the wrist. In the current covid-19 pandemic, with the emergence of vaccines, health professionals have been required to perform the manual homogenization very frequently, and the repetitive execution of this task is prone to induce damage to the articulation [19], [20].

In this paper, the proposed solution presents the development of a low-cost homogenizer to be used during the manipulation of Pfizer-BioNTech covid-19 vials. While the system is working with ten vials, the pharmacist can be redirected to other tasks avoiding the participation in repetitive movements since each vial should be gently turned 180 degrees twenty times, as described in section 2 . Furthermore, the system holds an infrared sensor that starts the homogenization process as soon as the operator passes the hand through it. Hence, there is no contact between the operator and the system reducing possible contamination and transmission. The specific use case of Pfizer-BioNTech covid-19 vaccine homogenization is demonstrated here but this concept can be applied to several medicines depending on the product characteristics. The proposed prototype and all the source files needed to assemble the system are provided further in this work, in order to replicate it as easily as possible. Experiments were conducted in both controlled and real environments, in a collaboration with the hospital pharmacy of Centro Hospitalar de Vila Nova de Gaia/Espinho, E.P.E., Portugal. We believe that this system will allow us to automate the homogenization process according to the specifications of each medicine. Moreover, the operator's ergonomics is taken into consideration reducing the effects caused by the repetitive handling of vials.

The structure of this paper is the following: after a brief introduction about the covid-19 global panorama and the work regarding the application of the homogenization process, section 2 describes the vial manipulation process. The architecture overview of the presented automated system is described in section 3 referring to the hardware components. In section 4, the mechanical design is addressed presenting the research methodologies used and the specifications of each of the designed 3D parts and their interconnections. Section 5 presents the results achieved and shows the experimental tests conducted in a real hospital environment. Finally, section 6 sums up the work with conclusions and discusses functionalities that may be interesting to add in the near future.

\section{COMIRNATYTM DISPERSION PROCEDURE}

The Pfizer-BioNTech covid-19 vaccine is a suspension for intramuscular injection administered as a course of two doses $(0.3 \mathrm{~mL}$ each) three weeks apart. Each vial is able to produce six vaccines syringes if correctly handled. According to the SmPC, provided by the European medicines agency (EMA) [21], the Comirnaty ${ }^{\mathrm{TM}}$ concentrate for dispersion for injection covid-19 mRNA vaccine (Pfizer-BioNTech covid-19) should follow a protocol for the vaccination procedure. The initial preparation step (related to this paper) is composed by two phases: thawing prior to dilution and the dilution. Regarding the first phase, frozen vials should be transferred to an environment of $2^{\circ} \mathrm{C}$ to $8^{\circ} \mathrm{C}$ to thaw. Then, it should be allowed the thawed vial to 
come to room temperature and gently invert it 10 times prior to dilution, as indicated on Figure 2 . The vials cannot be shaken. The thawed dispersion may contain white to off-white opaque amorphous particles.

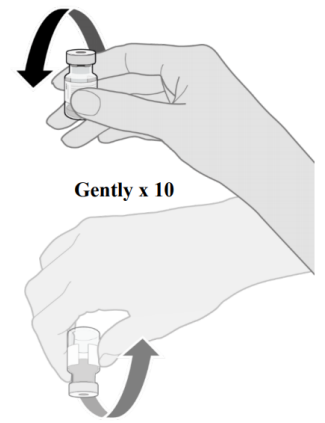

Figure 2. Gentle movement proposed by the SmPC [21]

On the second phase, the thawed vaccine must be diluted in its original vial with sodium chloride $9 \mathrm{mg} / \mathrm{mL}(0.9 \%)$ solution for injection. The vial pressure should be equalised before removing the needle from the vial stopper. Then, the diluted dispersion must be, again, gently inverted for 10 times (Figure 2). The proposed low-cost system will help the pharmacist and technician during the repetitive movement (gently inverted for 10 times).

\section{SYSTEM ARCHITECTURE}

The proposed system architecture is presented in Figure 3. It is composed by an Infrared motion detector that triggers the movement of the vials. An embedded system is used to control the servomotor that is coupled to the vials holder. The following subsections address the hardware and software developments exhaustively.

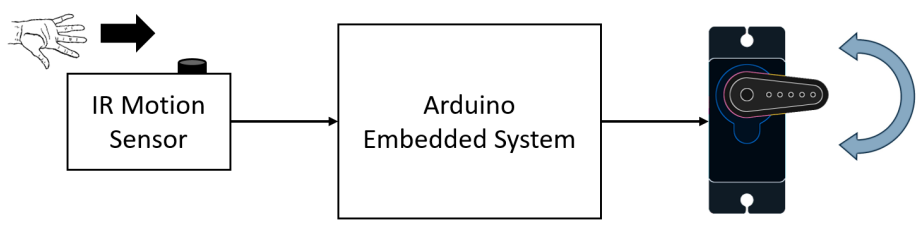

Figure 3. System architecture. The motion detection sensor (left), the arduino based embedded system (centre) and the servomotor that is coupled to the device (right)

\subsection{Hardware}

The developed system resorts on well-known and low-cost components that can be acquired from several robotics hobbyist stores. The detection of the pharmacist's hand that will start the inversion of the vials is performed by the obstacle detector. It is composed by an emitter and receiver of infrared (IR) light (Near infrared region $-700 \mathrm{~nm}$ to $1400 \mathrm{~nm}$ ). When the IR transmitter emits radiation, it reaches the object and some of the radiation is reflected back to the IR receiver. Based on the intensity of the reflected radiation reaching the IR receiver, the output of the sensor is defined [22]. The module has a digital output whose sensitivity can be adjusted with a potentiometer. Infrared light has been used for a long time in sensory applications [23].

The vials are forced to move by the servomotor shaft. It is also a well-known and low-cost component that can be acquired in stores that commercialise products dedicated to model air-planes and remote-control models. In this work, the HK15138 servomotor was used. The servomotor is powered by the $5 \mathrm{~V}$ provided by the system and can be controlled by a pulse width modulation (PWM) signal implemented through the Servo library included in the arduino integrated development environment (IDE), as explained in subsection 3.2 [24]. Servomotors have already been around for some decades and they are extremely popular among roboticists due to their simple control technique, reliability and low cost [25]. A servomotor is controlled by sending pulses through the signal line. The frequency of the control signal should be $50 \mathrm{~Hz}$, in other words, a pulse should 
occur every $20 \mathrm{~ms}$ [26]. The width of the pulse determines the angular position of the servomotor. For the used servomotor, pulses with $1 \mathrm{~ms}$ duration correspond to the 0 degrees position whereas those with a duration of $2 \mathrm{~ms}$ correspond to the 180 degrees position. For middle angles, the duration of the pulse should be interpolated. Examples of 0, 90 and 180 degrees are shown in Figure 4 [27].

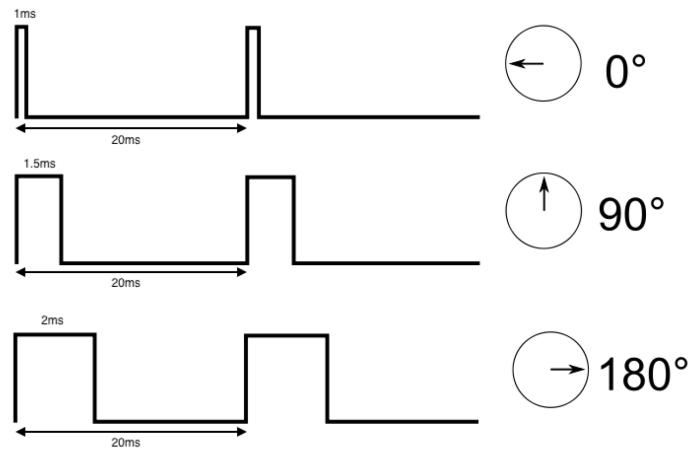

Figure 4. Examples of PWM signals to control the servomotor shaft position [27] at 0, 90, and 180 degrees

The embedded system that controls the entire system is based on the well-known Arduino Uno [28]. It is a development board with an IDE that allows to create programs in $\mathrm{C}$ and $\mathrm{C}++$ language. It owns an ATMega328 8-bit microcontroller which can be used to execute the developed software (addressed in subsection section 3.2) stored in its flash non-volatile program memory [29]. It owns several analog inputs and digital general-purpose input/output pins, six of them with PWM capability [30]. The used servomotor PWM signal is generated with one of these PWM pins (pin 6). The general schematic of the proposed system is presented in Figure 5. As it can be seen, the entire system is easily replicated.

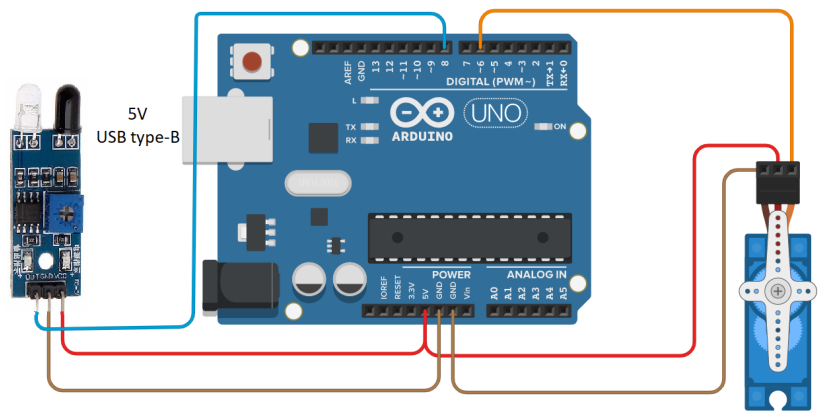

Figure 5. Electrical schematic of the entire system

The supply voltage for the servomotor and IR sensor is branched from the main Arduino board. With a type-B connector, it is powered by an USB common $5 \mathrm{~V}$ mobile charger that supports, at least, $1 \mathrm{~A}$ current. The IR sensor and the Arduino can be placed inside a closed case with holes for the IR emitter and receiver diodes allowing a contactless command for an easy disinfection and cleaning with sterile isopropyl alcohol (IPA).

\subsection{Software}

The software was developed on Arduino IDE in C language [31]. A flowchart of the proposed code is presented on Figure 6. It uses the Servo library from Arduino, a well-known library used in this type of projects.

The $\operatorname{setup}()$ function will configure the pin 8 as input and its pull-up resistor is activated internally in the microcontroller. The pin 6 is attached to the servomotor and its position is set to zero degrees. The $\operatorname{loop}()$ function will be detecting the pharmacists hand and then will trigger the movement. A simple switch debouncing software is introduced in order to avoid false detections due to unintentionally moving the hand 
above the sensor. A wait cycle of $300 \mathrm{~ms}$ is enough to perform this task. After that, the servomotor starts its clockwise and counter clockwise movements.

Since there is no feedback of the shaft position (a low-cost servomotor was used), a wait cycle was introduced to ensure the servomotor reaches the final angle. The same approach is used for the counter-clockwise movement. A for cycle is used to perform the number of the desired iterations. At the end, the hand detector is used to start another set of movements, allowing the user to change the vials holder for a new one to dilute/manipulate with saline solution.

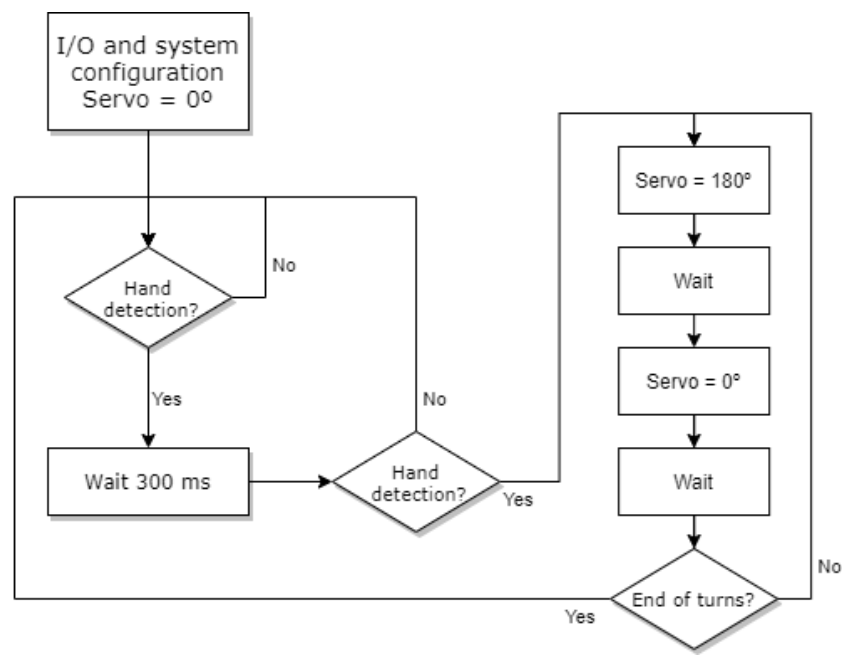

Figure 6. Flowchart for the software description

\section{MECHANICAL DESIGN}

The research methodologies and 3D design and modelling of the system are covered in this section. Initially, a kinematic study is conducted in order to find the adequate rotating axis of the vials. Afterwards, the system is 3D designed and modelled addressing the main mechanical parts as well as their interconnections [32]. Finally, the inertial moments and the time spent by the system to perform a half turn are specified.

\subsection{Definition of the rotation axis}

A kinematic study was conducted to validate the proposed approach. The vial measurements are detailed in Figure 7 where:

$-h=32 \mathrm{~mm}$;

$-\phi_{1}=16 \mathrm{~mm}$

$-\phi_{2}=14 \mathrm{~mm}$

$-h_{L 1}=2.7 \mathrm{~mm}$ (height of liquid on the first homogenization);

- $h_{L 2}=15 \mathrm{~mm}$ (height of liquid on the second homogenization);

$-m_{v}=4.1 \mathrm{~g}$ (mass of an empty vial);

- $m_{1}=0.4 \mathrm{~g}$ (mass of the liquid on the first homogenization);

$-m_{2}=2.2 \mathrm{~g}$ (mass of the liquid on the second homogenization, after introducing $1.8 \mathrm{~g}$ of sodium chloride);

In order to calculate the center of mass for the first and the second homogenization phases, where the vials are, respectively, almost empty or with liquid, the vial was assumed to be a cylinder with dimensions radius $\phi_{1}$ and height $h$. The center of mass will fit along the longitudinal axis of the cylinder. Considering the base of the vial the y coordinate zero, the height of the center of mass for the first homogenization $\left(C M_{Y 1}\right)$ can be calculated as expressed on (1), equalling $14.70 \mathrm{~mm}$ (blue circle on Figure 7) for $x$ assuming the value of 1 .

$$
C M_{Y x}=\frac{\frac{h}{2} \cdot m_{v}+\frac{h_{L x}}{2} \cdot m_{x}}{m_{v}+m_{x}}
$$


Regarding the height of the center of mass for the second homogenization process $\left(C M_{Y 2}\right)$, the result was $13.03 \mathrm{~mm}$ (red circle on Figure 7) for $x$ assuming the value of 2. To account for both cases, the height of the center of mass of the vials was considered the average between $C M_{Y 1}$ and $C M_{Y 2}$, resulting in $13.87 \mathrm{~mm}$, which defined the axis of rotation.

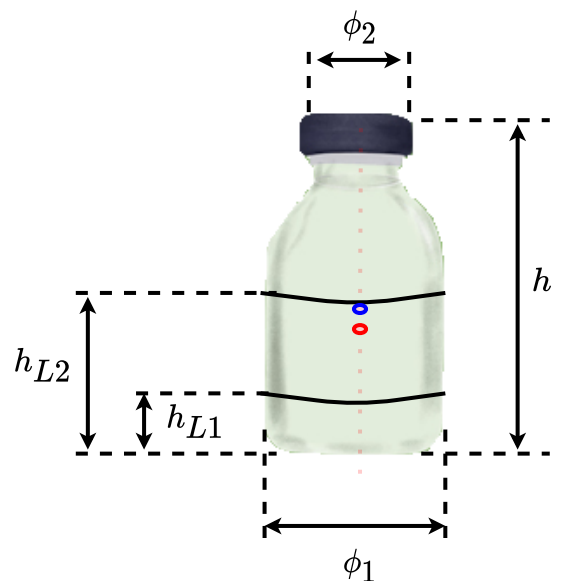

Figure 7. Schematic of the Pfizer-BioNTech covid-19 vaccine vial measurements. Blue and red circles represent, respectively, the center of mass for an empty vial case and with liquid

\subsection{System 3D modelling}

All the parts were designed [33] and 3D printed with polylactic acid (PLA), a common filament used on $3 \mathrm{D}$ printers, since it is economically produced from renewable resources, such as corn. PLA is a type of bioplastic, which is plastic derived from plant or biological material instead of oil. Even though its properties are modified at high temperatures, the presented use case environment temperature is stable and low enough to avoid this problem. The mechanical components were developed on the OpenSCAD software. OpenSCAD is a free software application for creating solid 3D CAD objects (it can be downloaded from [34]). It is a script-only based modeller that uses its own description language [35]. The mechanical parts are composed by 3 components: a support base $\left(b_{s b}\right.$ in Figure 8$)$ that can be fixed by six M3 screws to a table or a planar support with the aim of sustaining the vaccine vials holder and housing the servomotor; a coupler $\left(b_{c p}\right.$ in Figure 8$)$ to mechanically connect the servomotor shaft to the vials holder; and a vials holder $\left(b_{h l}\right.$ in Figure 8$)$ to support ten vaccine vials.

The connections between the three components of the system are also depicted in Figure 8 . Both the vials holder and the support base components are made of the same PLA material. In order to avoid the friction of the link $j_{1}$ and, consequently, increase the materials longevity [36], it is advisable to include a frictionless material on the support tower. The controlled room temperature where this system is applied guarantees that the PLA deformation does not occur. Moreover, some topics were carefully taken into consideration during the development of the proposed system. The rotation movement between the vials holder and the base was firstly designed to hold a bearing ring. However, this would increase the complexity, the cost and the disinfection process needs. Therefore, it was replaced by a friction-less material (polypropylene-commonly used in syringes) that can sustain the axis of the vials holder and the base while it turns. The results for more than one thousand rotations did not show any wear for these components. Furthermore, the insertion and removal of the vials from the vials holder may lead to the deformation or damage of the latter. Despite that, the contact will be made between the printed plastic and the glass material of the vials which has very low friction resulting in minimal deterioration. Nevertheless, for both described situations, a stronger material such as injected plastic or metal can be used. The connection part between the vials holder shaft and the coupler $\left(j_{2}\right)$ should be tighten by four M2 screws. The coupler was designed with four equally spaced protrusions to properly fit the quad-arm servo horn of the servomotor's shaft. The latter performs a rotating movement in the range $[0,+180]$. A deliberated slack was introduced in this link $\left(j_{3}\right)$ in order to allow for an easy and time efficient replacement of the vials holder.

The holder can house up to ten vials and move them simultaneously. This constant of ten was achieved 
as a compromise between the time required to introduce the vials, the homogenization time and the optimisation of the number of vials that the pharmacist can prepare simultaneously. Figure 9 presents the developed 3D model that can hold ten vials for homogenization, focusing the $11 \mathrm{~mm}$ diameter bottleneck click system, which was designed for an easy and fast manipulation (insertion and removal) of the vial by the pharmacist. This design should be replicated for each vial support.

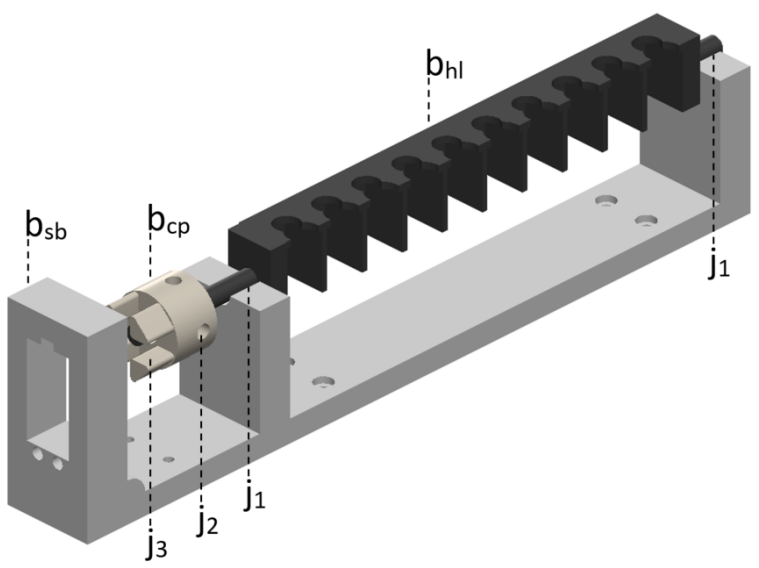

Figure 8. 3D CAD schematic of the proposed system, which comprises a support base $\left(b_{s b}\right)$, a coupler $\left(b_{c p}\right)$, a vials holder $\left(b_{h l}\right)$, and their respective links $\left(j_{1}\right.$ links $b_{s b}$ to $b_{h l}, j_{2}$ links $b_{c p}$ to $b_{h l}$ and $j_{3}$ links the servomotor shaft to $b_{c p}$ )

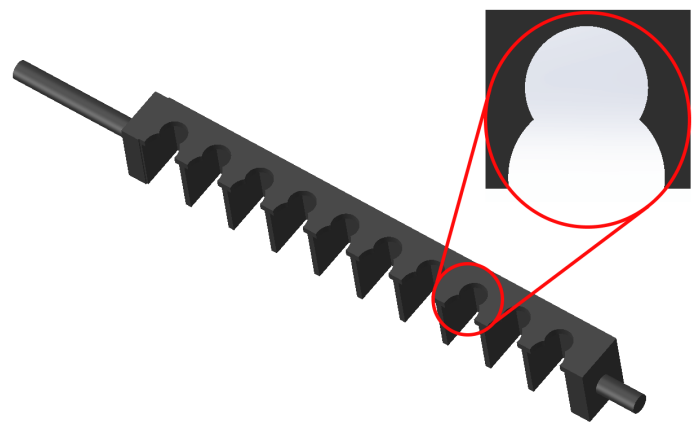

Figure 9. 3D CAD of the vials holder focusing the bottleneck click system for easy insertion and removal of the vaccine vials

\subsection{Definition of the angular speed of the servomotor}

For a dynamic analysis, the mass and dimensions of the moving parts were measured as follows. The coupler can be represented as a cylinder of $15 \mathrm{~mm}$ of radius $\left(r_{c p}\right)$ weighing $8 \mathrm{~g}\left(m_{c p}\right)$, whereas the vials holder can be approximated as a cylinder of $10 \mathrm{~mm}$ of radius $\left(r_{h l}\right)$ weighing $35 \mathrm{~g}\left(m_{h l}\right)$, and the weight of the support base is $110 \mathrm{~g}\left(m_{s b}\right)$.

The inertia [37] of the coupler $\left(I_{c p}\right)$ can be calculated as expressed on (2), being equal to $9.00 E-7$ $\mathrm{kg} \cdot \mathrm{m}^{2}$. The same approach can be applied to the vials holder, being its inertia $\left(I_{h l}\right)$ equal to $1.76 E-2 \mathrm{~kg} \cdot \mathrm{m}^{2}$.

$$
I_{x}=\frac{1}{2} \cdot m_{x} \cdot r_{x}^{2}
$$

The inertia of each vial $\left(I_{v}\right)$ is estimated to be $3.83 E-6 \mathrm{~kg} \cdot \mathrm{m}^{2}$, according to (3), which assumes that each vial is a cylinder rotating perpendicularly to the long axis of the vials holder.

$$
I_{v}=\frac{1}{2} \cdot\left(m_{v}+m_{2}\right) \cdot\left(3 \phi_{1} / 2+h^{2}\right)
$$


The total inertia $\left(I_{T}\right)$ that the servomotor will have to deal with can be expressed as the sum of all previous inertia values, as given by (4), summing up to $1.76 \mathrm{E}-2 \mathrm{~kg} \cdot \mathrm{m}^{2}$.

$$
I_{T}=I_{c p}+I_{h l}+10 \cdot I_{v}
$$

From the characteristics of the servomotor, a maximum torque $\left(T_{m}\right)$ of $0.38 \mathrm{~N} . \mathrm{m}$ and a maximum speed $\left(\omega_{M A X}\right)$ of $4.98 \mathrm{rad} / \mathrm{s}$ are available when supplied at $4.8 \mathrm{~V}$. The maximum angular acceleration $\left(\alpha_{M A X}\right)$, which can be calculated by $T_{m} / I_{T}$, is $21.5 \mathrm{rad} / \mathrm{s}^{2}$. This value allows to find the time $\left(t_{\text {tran }}\right)$ to reach $\omega_{M A X}$, being calculated as $\omega_{M A X} / \alpha_{M A X}$, taking the value of $230 \mathrm{~ms}$. Having the $\alpha_{M A X}$ and the $t_{\operatorname{tran}}$ values, the angle $\theta$ that is rotated during $t_{\text {tran }}$ is calculated as expressed on (5) resulting in 0.576 rads.

$$
\theta=\frac{1}{2} \cdot \alpha_{M A X} \cdot t_{t r a n}^{2}
$$

Assuming the acceleration and deceleration are symmetric, for a half turn (as required), 1.99 rads are still missing by calculating $\pi-2 \cdot 0.576$. Rotating 1.99 rads at $\omega_{M A X}$ takes $400 \mathrm{~ms}$. Hence, a total of $862 \mathrm{~ms}$ would be needed to turn $\pi$ rad with initial and final angular speeds of $0 \mathrm{rad} / \mathrm{s}$. However, in this case study, the servomotor was supplied with $5.0 \mathrm{~V}$ instead of $4.8 \mathrm{~V}$, slightly reducing the $t_{\text {tran }}$ value, taking a total of $800 \mathrm{~ms}$ to perform a half turn.

\section{RESULTS AND DISCUSSION}

The proposed and developed homogenizer was tested in the Centro Hospitalar de Vila Nova de Gaia/Espinho, E.P.E. (CHVNG/E, E.P.E.), Portugal. A thousand vials were processed to prepare more than four thousand covid-19 vaccines to be administrated to health professionals. Figure 10 presents the assembled prototype inside a laminar flow chamber in a clean room ready for the process.

As already mentioned, one of the advantages of the proposed homogenizer is the capability of helping these healthcare workers to perform parallel processes, in other words, giving them the chance to do other tasks while the homogenizer moves the vaccine vials. This situation is illustrated in Figure 11.

The introduction of the $300 \mathrm{~ms}$ delay was very useful since no unintentional movement of an arm or a hand through the sensor triggered the system activation, resulting in none unwanted process start.

The sequence of the preparation for the Comirnaty ${ }^{\mathrm{TM}}$ concentrate for dispersion for injection covid-19 mRNA vaccine (Pfizer-BioNTech covid-19) is presented on Figure 12. Sub-pictures (a) to (f) show the main interactions with the system. The operator started by inserting the vials into the holder until all of the ten spaces were filled (image a). After that, the holder (black) was placed in the homogenizer base (white support). Then, the user triggered the homogenization process through the contactless sensor and the first homogenization phase was started (image b). While the vials were in the homogenization process, the sodium chloride measure was made (image c). As soon as the homogenization process finished, the pharmacist introduced the sodium chloride inside each vial (image d) and, then, the holder returned to the homogenizer for the second and last homogenization (image e). Finally, each dose of vaccine was withdrawn to be administrated (image f). As main result, the developed system allowed to speed-up the preparation of the covid-19 vaccines and also avoided musculoskeletal disorders of the hospital pharmacy staff. Besides, the hospital pharmacy of the Centro Hospitalar de Vila Nova de Gaia/Espinho, E.P.E. (CHVNG/E, E.P.E.), where this system was introduced, recognised it as an important component during the preparation of the vaccines.

The used components can be acquired in common electronics stores. Basically, this system requires some 3D printed parts, an Arduino and an Infrared motion detector instead of a mechanical press button, in order to start the homogenization process in a contactless manner, avoiding contamination. It is possible to replicate this system since all the required files and source code are available.

As a final remark, the hospital was recognised by the national healthcare system as the one achieving the highest ratio between the number of vaccines administrated and the number of vials. The additional time that the operator had while the system was homogenizing the vials, may have contributed to a more adequate preparation. After a short inquiry, all the technicians congratulated the prototype validating the proposed approach. 


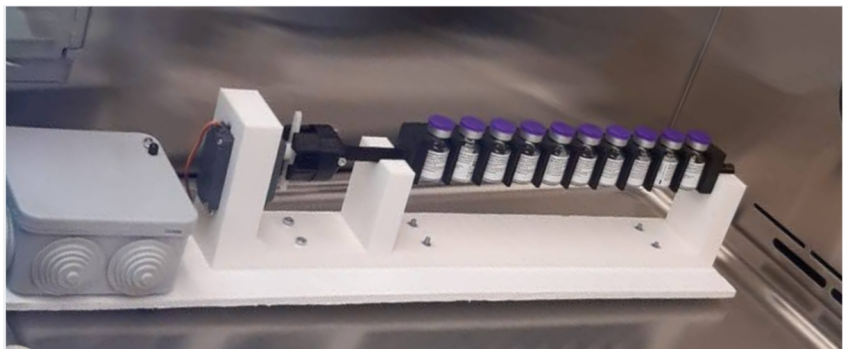

Figure 10. The proposed homogenizer in a real hospital environment (at hospital pharmacy of Centro Hospitalar de Vila Nova de Gaia/Espinho, E.P.E) with ten Pfizer-BioNTech covid-19 vaccines vials

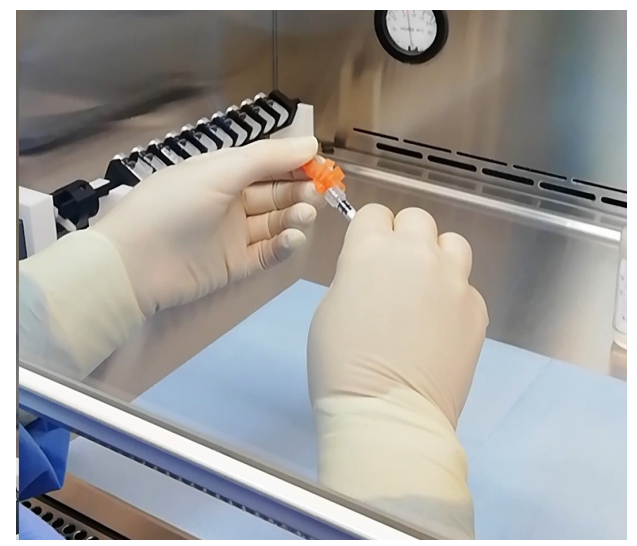

Figure 11. A parallel process is being conducted while the developed homogenizer mixes the vials, reducing the operation time
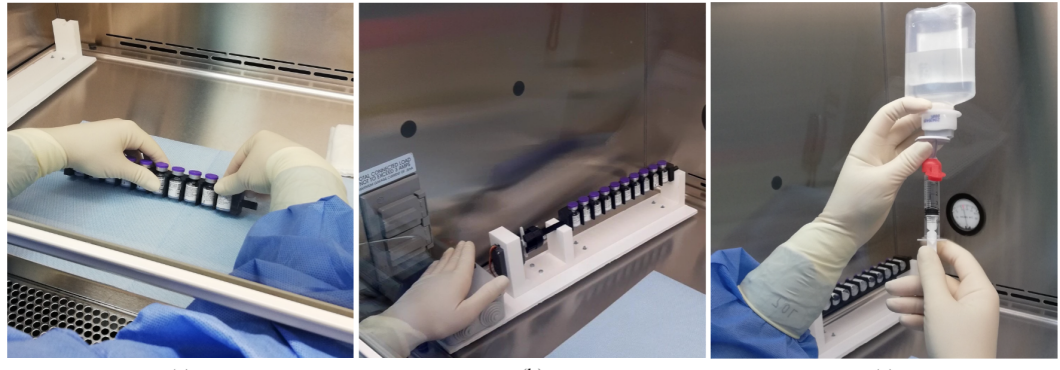

(b)
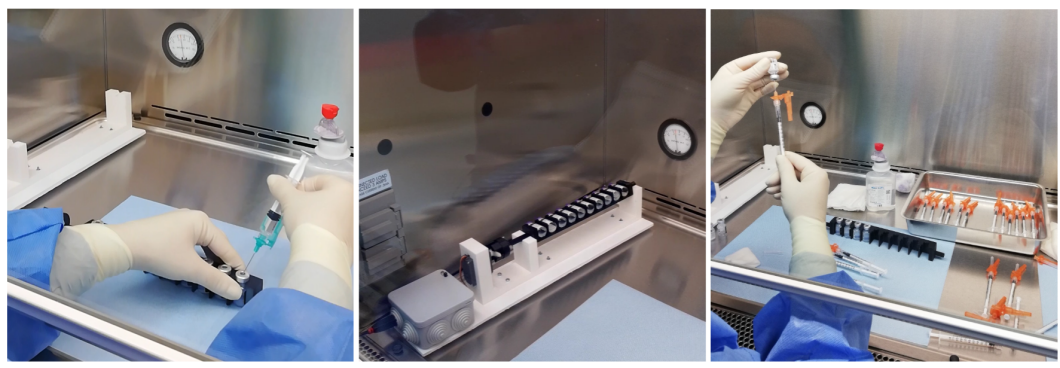

Figure 12. Snapshots taken during the preparation phases: (a) Inserting the vials into the holder,

(b) Triggering the homogenization process through the contactless sensor, (c) Measuring the sodium chloride while vials are in the homogenization process, (d) Introducing the sodium chloride inside each vial, (e) Second homogenization is performed, (f) Loading the syringes with the vaccine to be administrated 


\section{CONCLUSION}

This paper presented a homogenizer for helping the hospital pharmacy department with the preparation of the Pfizer-BioNTech covid-19 vaccine, which requires a specific manipulation by the pharmacists before its administration. There were three main objectives to fulfill with the introduction of this prototype. The first one was to avoid the risk that this repetitive process poses to the ergonomics of the hospital pharmacy staff. The second one was to minimize the contact with the developed system to reduce the contamination, which has been achieved using a contactless Infrared sensor. Finally, the third one was to redirect the healthcare workers to other tasks while the homogenizer is operating. The proposed system allows to prepare sixty vaccines each iteration, since each vial produces six vaccines. The aim of this paper was to disseminate a project relevant for the current pandemic which can be replicated with some basic components such as an Arduino Uno, a servomotor, some 3D printable parts and the source files provided by the authors. It is fast and easy to construct and it is helpful for the preparation of this type of vaccines that must be administrated in a large scale. The operators recognised this system as an important component during the preparation of the vaccines. Until now, the developed device homogenized more than eight thousand Pfizer-BioNTech covid-19 vaccines according to the summary of product characteristics, automating the repetitive tasks and, consequently, redirecting the healthcare workers to other important tasks. As future work, an adjustable speed of the homogenizer can be introduced, as well as a configurable duty-cycle of the operation and the number of cycles. The holder can be also adjusted to other sizes of vials.

\section{ACKNOWLEDGEMENT}

The developers of this system thank the pharmacy department of the Centro Hospitalar de Vila Nova de Gaia/Espinho, E.P.E for supporting the implementation, tests and validation of the proposed system.

This work is financed by National Funds through the Portuguese funding agency, FCT - Fundação para a Ciência e a Tecnologia within project UIDB/50014/2020.

\section{REFERENCES}

[1] L. Amelia and R. A. Syakurah, "Analysis of public search interest towards immune system improvement during the COVID-19 pandemic using Google Trends," International Journal of Public Health Science (IJPHS), vol. 9, no. 4, 2020, pp. 414-420, doi: 10.11591/ijphs.v9i4.20518.

[2] World Health Organization website, accessed on 20 Jan 2021. [Online] available: https://www.who.int/emergencies/diseases/novel-coronavirus-2019.

[3] N. Budiyanta, L. Wijayanti, W.W Basuki, H. Tanudjaja and V. Budi Kartadinata, "The development of healthcare mobile robot for helping medical personnel in dealing with COVID-19 patients," Indonesian Journal of Electrical Engineering and Computer Science, vol. 22, no. 3, 2021, pp. 1379-1388, doi: 10.11591/ijeecs.v22.i3.pp1379-1388.

[4] S. J. Alley, et al., "As the Pandemic Progresses, How Does Willingness to Vaccinate against COVID-19 Evolve?," International Journal of Environmental Research and Public Health , vol. 18, no. 2, p. 797, 2021, 10.3390/ijerph18020797.

[5] D. E. Speiser and M. F. Bachmann, "COVID-19: Mechanisms of Vaccination and Immunity," Vaccines, vol. 8, no. 3, p. 404, 2020, doi: 10.3390/vaccines8030404.

[6] R. H. Dodd, et al., "Concerns and motivations about COVID-19 vaccination," Comment, Lancet Infect Diseases, vol. 21, no. 2, pp. 161-163, 2021, doi: 10.1016/S1473-3099(20)30926-9.

[7] E. Callaway, "What Pfizer's landmark COVID vaccine results mean for the pandemic," Nature, 2020, doi: 10.1038/d41586-020-03166-8.

[8] A. Mundra and B. S. Garg, "Prospects of vaccine against COVID-19," Indian Journal of Community Medicine, vol. 45, no. 4, pp. 391-395, 2020, doi: 10.4103/ijcm.IJCM_849_20.

[9] E. A. Zelentsovaab, V. V. Yanshole and Y. P. Tsentalovich, "A novel method of sample homogenization with the use of a microtome-cryostat apparatus," RSC Advances, Royal Society of Chemistry, vol. 9, no. 65, pp. 37809-37817, 2019, doi: 10.1039/C9RA06808B.

[10] Y. Xiong, V. Shapaval, A. Kohler and P. J. From, ”A Laboratory-Built Fully Automated Ultrasonication Robot for Filamentous Fungi Homogenization," SLAS TECHNOLOGY: Translating Life Sciences Innovation, vol. 24, no. 6, pp. 583-595, 2019, doi: 10.1177/2472630319861361.

[11] Y. Xiong, V. Shapaval, A. Kohler, J. Li and P. J. From, ”A Fully Automated Robot for the Preparation 
of Fungal Samples for FTIR Spectroscopy Using Deep Learning,” in IEEE Access, vol. 7, pp. 132763132774, 2019, doi: 10.1109/ACCESS.2019.2941704.

[12] Electron Microscopy Sciences, Rotamix ${ }^{\mathrm{TM}}$, accessed on 18 Jan 2021. [Online]. Available: https://www.fishersci.com/shop/products/rotamix-rkvs/50996362.

[13] Labnet, Mini LabRoller Rotator with Rotisserie, accessed on 23 Jan 2021. [Online]. Available: https://www.fishersci.pt/shop/products/international-mini-shaker-lab-roller/11895953.

[14] Benchmark Scientific, Rotating Mixer, accessed on 22 Jan 2021. [Online]. Available: https://lab.equipment/r5010-rotating-mixer-from-benchmark-scientific.

[15] Meditech, Blood Mixer, accessed on 20 Jan 2021. [Online]. Available: http://newmeditech.com/products/blood-collection/blood-mixer/.

[16] PharmaSystems, MediMix Vial Reconstitution Mixer, accessed on 21 jan 2021. [Online]. Available: https://www.pharmasystems.com/index.php?route=product/product\&product_id=2645.

[17] Unico, Tube Rocker, accessed on 21 Jan 2021. [Online]. Available: https://www.unicosci.com/tuberocker-l-ttr100.html.

[18] Center for Disease Control and Prevention: Work-related musculoskeletal disorders and ergonomics, 2018, accessed on 17-jan-2021. [Online]. Available: https://www.cdc.gov/workplacehealthpromotion/health-strategies/musculoskeletal-disorders/index.html.

[19] E. Wild, S. G. Gerberich, K. Hunt and K. Coe, "Analyses of Wrist Injuries in Workers Engaged in Repetitive Tasks," AAOHN JOURNAL, vol. 35, no. 8, pp. 356-366, 1987, doi: 10.1177/216507998703500805.

[20] Y.-L. Chen and Y.-S. Ou, "A case study of Taiwanese custom-beverage workers for their musculoskeletal disorders symptoms and wrist movements during shaking task," International Journal of Industrial Ergonomics, vol. 80, p. 103018, 2020, doi: 10.1016/j.ergon.2020.103018.

[21] European Medicines Agency, accessed on 24 Jan 2021. [Online]. Available: https://www.ema.europa.eu/en.

[22] A. A. Abbood, Q. M. Shallal and M. Fadhel, 'Internet of things (IoT): a technology review, security issues, threats, and open challenges," Indonesian Journal of Electrical Engineering and Computer Science, vol. 20, no. 3, pp. 1685-1692, 2020, doi: 10.11591/ijeecs.v20.i3.pp1685-1692.

[23] K. Iniewski, Smart Sensors for Industrial Applications (Devices, Circuits, and Systems), CRC-Press:USA, 2017, doi: 10.1201/b14875.

[24] Muhardika and Syafii, "Design of arduino-based loading management system to improve continuity of solar power supply," Indonesian Journal of Electrical Engineering and Computer Science, vol. 20, no. 3, pp. 1677-1684, 2020, doi: 10.11591/ijeecs.v20.i3.pp1677-1684.

[25] D. Aragon-Jurado, A. Morgado-Estevez and F. Perez-Peña, "Low-Cost Servomotor Driver for PFM Control," Sensors, vol. 18, no. 1, p. 93, 2018, doi: 10.3390/s18010093.

[26] Y. Samkari, K. Guedri, M. Oreijah, S. Munshi and S. Azam, "Designing and testing of a smart firefighting device system (LAHEEB)," International Journal of Robotics and Automation (IJRA), vol. 9, no. 2, pp. 143-152, 2020, doi: 10.11591/ijra.v9i2.pp143-152.

[27] R. Firoozian, Servo Motors and Industrial Control Theory, Springer, 2014, doi: 10.1007/978-3-31907275-3.

[28] I. A. Taha and H. M. Marhoon, "Implementation of Controlled Robot for Fire Detection and Extinguish to Closed Areas Based on Arduino," TELKOMNIKA (Telecommunication Computing Electronics and Control), vol. 16, no. 2, pp. pp. 654-664, 2018, doi: 10.12928/TELKOMNIKA.v16i2.8197.

[29] B. M. Amine, C. F. Zohra, H. Ilyes, A. Lahcen and A. Tayeb, ”Smart Home Automation System Based on Arduino," International Journal of Robotics and Automation (IJRA), vol.7, no.4, pp. 215-220, 2018, doi: 10.11591/ijra.v7i4.pp215-220.

[30] T. Gross, K. Anderson and N. Tsuchiya, "Programmable Automation Controller Mechatronic Experiment," International Journal of Robotics and Automation (IJRA), vol. 6, no. 1, pp. 39-48, 2017, doi: 10.11591/ijra.v6i1.pp39-48.

[31] A. Vidstrom, The Vidstrom Labs Guide to Advanced Arduino Internals for the Uno and Mega 2560, Vidstrom Labs, 2020.

[32] G. Qaryouti, A. R. Salbad, S. A. Tamimi, A. Almofleh, W. A. Salah and Q. Jabe, ”Design and implementationof a three dimensions(3D) printerfor modelingand pre-manufacturing applications," International Journal of Electrical and Computer Engineering (IJECE), vol. 9, no. 6, pp. 4749-4757, 2019, doi: 10.11591/ijece.v9i6.pp4749-4757. 
[33] Z. F. Z. Abidin and M. N. O. Zahid, "Real time object customization in CAD system," Indonesian Journal of Electrical Engineering and Computer Science, vol. 15, no. 2, pp. 1066-1075, 2019, doi: 10.11591/ijeecs.v15.i2.pp1066-1075.

[34] OpenSCAD website, accessed on 17 Jan 2021. [Online]. Available: https://www.openscad.org/.

[35] A. Williams, OpenSCAD for 3D Printing, 1st Edition, 2014.

[36] I.-T. Maries, C. Vilau, M. S. Pustan, C. Dudescu and H. G. Crisan, ”Determining the tribological properties of different 3D printing filaments," IOP Conference Series: Materials Science Engineering, vol. 724, p. 012022, 2020, doi: 10.1088/1757-899X/724/1/012022.

[37] E. Nelson, Engineering Mechanics Statics, McGraw-Hill, 2010.

\section{BIOGRAPHIES OF AUTHORS}

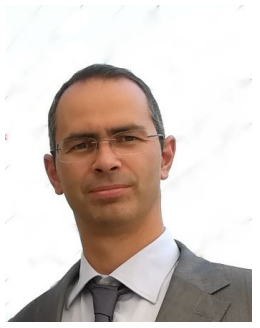

José Lima received the M.Sc. and PhD in Electrical and Computer Engineering on Faculty of Engineering of University of Porto, Portugal in 2001 and 2009. He is a Professor in the Electrical Engineering Department at Polytechnic Institute of Bragança since 2002. He is also a vice coordinator of the Research Centre in Digitalization and Intelligent Robotics, and Member of the coordination council of the Centre for Robotics in Industry and Intelligent Systems group of the INESC TEC (Institute for Systems and Computer Engineering of Porto, Portugal). He has published more than 100 papers in international scientific journals and conference proceedings. He participated in some national, FP7 and H2020 funded projects such as Produtech, Grace, Arum, Carlos, Stamina and ColRobot. Further info on his homepage: https://orcid.org/0000-0001-7902-1207

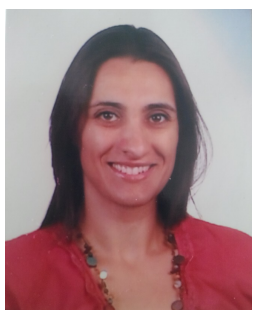

Luísa Rocha received the PharmD and M.Sc. in Pharmaceutical Sciences on Faculty of Pharmacy of University of Porto, Portugal in 2000 and 2011. She had her Specialization in Hospital Pharmacy by the Portuguese Pharmacy Council - Portugal, in 2011. She worked as a Community Pharmacist from May 2000 to June 2003, and joined the Centro Hospitalar do Nordeste E.P.E. - U. H. Bragança in July 2003 as a Hospital Pharmacist, where her main ativities were validation of medical prescriptions, dispensing medicines and patient counseling in ambulatory care. She was also the responsible for the Hospital Pharmacy Department and a member of Pharmacy and Hospital Therapy Committee and an advisory member of Hospital Infection Control Committee until June 2007. She joined the Hospital Pharmacy Department in Centro Hospitalar de Vila Nova de Gaia in June 2007, and integrated the compounding sterile products for patients including Total Parenteral Nutrition and Chemotherapy areas, as well as participation in several phase II, III and IV clinical trials. Currently she is the Hospital Pharmacy Department Director and also a member of Pharmacy and Hospital Therapy Committee. She has been responsible for several curricular internships and presentations in national conferences.

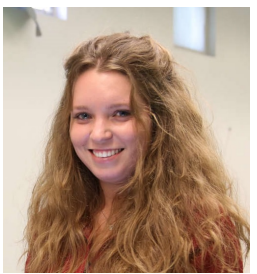

Cláudia Rocha received the M.Sc. in Bioengineering, specialization in biomedical engineering, from the Faculty of Engineering of University of Porto, Portugal in 2016. She joined the Centre for Robotics in Industry and Intelligent Systems at INESC TEC on 2016 as a researcher, participating in various projects, such as RADAR, an autonomous robot for disinfection in hospital environment; $\mathrm{RDH}$, a robot for distribution in hospitals; and PNEUMA, a low-cost and easy to assemble ventilator with a self-inflating bag that is supporting Portuguese hospitals during the COVID-19 pandemic. Her roles comprise 3D modelling, mobile robotics, automation and web interfaces.

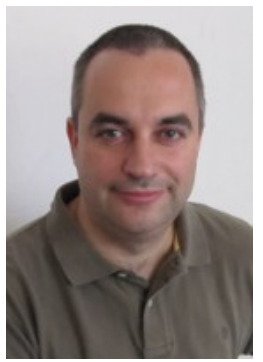

Paulo Costa received the M.Sc. and $\mathrm{PhD}$ in Electrical and Computer Engineering on Faculty of Engineering of University of Porto, Portugal in 95 and 2000. He joined Faculty of Engineering of University of Porto in 1992, and currently he is a Professor in the Electrical Engineering Department. $\mathrm{He}$ is also a senior researcher in Centre for Robotics in Industry and Intelligent Systems group of the INESC-TEC (Institute for Systems and Computer Engineering of Porto, Portugal). He has published more than a hundred papers in international scientific journals and conference proceedings. In addition, he participated in many autonomous mobile robotics competitions. Moreover, his research interests are in the field of robotics and automation: simulation, path planning, artificial vision, mobile robot localization and navigation, obstacle avoidance and perception. He participated in some national, International and H2020 funded projects such as PRODUTECH SIF, ScalABLE4.0 and CARLoS.

Int J Rob \& Autom, Vol. 10, No. 4, December 2021 : 340 - 352 